\title{
Broadband negative-index surface-waves on arrays of capped helices
}

\author{
P. Petrov $\odot,{ }^{*}$ A. P. Hibbins $\odot$, and J. R. Sambles $\odot$ \\ Centre for Metamaterial Research and Innovation, University of Exeter, Exeter, EX4 4QL, United Kingdom
}

N. Aboulkhair॰

Centre for Additive Manufacturing (CfAM), Faculty of Engineering, University of Nottingham, NG8 1BB, United Kingdom and Additive Manufacturing Laboratory, Advanced Materials Research Centre (AMRC), Technology Innovation Institute (TII), Building B04C, Masdar City, Abu Dhabi, P.O.Box 9639, United Arab Emirates

\author{
E. Saleh $\odot$ \\ University of Leeds, Faculty of Engineering, LS2 9JT, Leeds, United Kingdom
}

(Received 17 June 2021; revised 25 August 2021; accepted 16 September 2021; published 19 November 2021)

\begin{abstract}
A novel metasurface comprised of capped helices arranged as a hexagonal array that supports a broadband near-isotropic negative-index microwave surface-wave is designed, manufactured, and experimentally characterized. The surface-mode dispersion is studied both numerically and experimentally with the operational band more than double the bandwidth of structures previously reported in the literature. Hence, it is shown how one may provide a structured surface that supports just a negative-index mode over a wide operational band with no forward wave being simultaneously excited.
\end{abstract}

DOI: 10.1103/PhysRevResearch.3.043129

\section{INTRODUCTION}

Since the start of metamaterials research, possibly the most known effect that has been observed in metamaterial structures is that of negative refractive index. Such materials were first theoretically proposed in 1904 by Lamb [1] who suggested the idea of "backward waves" in reference to pressure waves. In the same year, Schuster [2] went on to quote Lamb's work and extended the idea to electromagnetic waves in his book "Theory of Optics." However, this mention of negative index of refraction by Schuster was little more than a minor comment. It was Veselago's work in 1968 that explored more fully the repercussions of electromagnetic negativeindex materials [3]. It has been shown that such materials should possess simultaneously negative electric permittivity $(\varepsilon)$ and magnetic permeability $(\mu)$. Waves that propagate in any medium characterized by a negative refractive index have momenta, wave vectors $(k)$, that are directed in the opposite direction to the energy flow $(S)$. The possibility of realizing such material was suggested in the works of Pendry and co-workers [4], who proposed a structure that combines negatively magnetically coupled meta-atoms [5] with metallic rods [6], where the electric coupling between them is also negative.

\footnotetext{
*pp386@exeter.ac.uk
}

Published by the American Physical Society under the terms of the Creative Commons Attribution 4.0 International license. Further distribution of this work must maintain attribution to the author(s) and the published article's title, journal citation, and DOI.
The experimental realization of negative-index propagation in these structures was demonstrated by Smith et al. [7]. Different geometries of meta-atoms have since been studied including split-ring resonators (SRRs) [7,8], swiss rolls [9], and helices [10]. Negative-index structures have been demonstrated for frequency ranges from microwave [7-10] to infrared $[11,12]$. One can also study the propagation of modes having negative mode index on structured surfaces; the "Sievenpiper mushroom" structure [13] is a well-known example of such a metasurface. While originally studied to offer an artificial magnetic conductor (AMC) boundary condition for antenna systems, it has also been demonstrated to support negative mode index surface-waves across a narrow bandwidth [14].

Negative-index structures may be useful for such applications as perfect lenses [5], cloaking devices [15], and in wavelength-division-multiplexing (WDM) systems based on negative-index metamaterials [16]. Moreover, metamaterials with negative index have already been implemented to increase the signal-to-noise ratio in magnetic resonance imaging. Although a variety of approaches and geometries have been proposed over the last decades, one general drawback of such structures has been their narrow operational bandwidths. The widest operational band, to the best of our knowledge, has been reported in $[17,18]$ where about $30 \%$ bandwidth compared to the resonant frequency has been achieved at $\mathrm{THz}$ and infrared regions. While in the microwave region the broadest reported operational band comprises only $20 \%$ of the resonant frequency [19]. Finally, the recently reported single-negative hyperbolic metamaterials that support broadband operation at optical frequencies [20] cannot be scaled to the microwave region. 
The operational band of a metamaterial surface may be calculated based on the coupling between elements. Consider a two-dimensional (2D) square array of coupled resonant elements. Each of these will have a self-impedance $Z_{0}$, and will be coupled to their nearest neighbors along both axes with magnetic and electric couplings $\kappa_{H}$ and $\kappa_{E}$, respectively. As a first approximation we will assume that the couplings to the next nearest (diagonal) neighboring elements is zero. Kirchhoff's equation for an element of the array at position $n$ along the $x$ axis and $m$ along the $y$ axis can be written in the following form:

$$
\begin{array}{r}
Z_{0} I_{n, m}+i \omega M\left(I_{\mathrm{n}-1, \mathrm{~m}}+I_{\mathrm{n}+1, \mathrm{~m}}+I_{\mathrm{n}, \mathrm{m}-1}+I_{\mathrm{n}, \mathrm{m}+1}\right) \\
+\frac{I_{\mathrm{n}-1, \mathrm{~m}}+I_{\mathrm{n}+1, \mathrm{~m}}+I_{\mathrm{n}, \mathrm{m}-1}+I_{\mathrm{n}, \mathrm{m}+1}}{i \omega K}=0 .
\end{array}
$$

This is similar to the one obtained in [21] for a linear (one-dimensional) array. Here $I_{n, m}$ is a current in element number $(n, m)$. Now, using the wave ansatz in the form $I_{n, m}=$ $I_{0} e^{i \omega t-i\left(n k_{x}+m k_{y}\right) a}$ (where $k_{x}$ and $k_{y}$ are the wave vectors along the $x$ and $y$ directions, respectively, and $a$ is the size of the unit cell) and applying the definition of electric and magnetic couplings introduced in [22], we obtain the following approximation for the dispersion equation for this metamaterial surface:

$$
f=f_{0} \sqrt{\frac{1-\kappa_{E}\left(\cos \left(\pi k_{x} a\right)+\cos \left(\pi k_{y} a\right)\right)}{1+\kappa_{H}\left(\cos \left(\pi k_{x} a\right)+\cos \left(\pi k_{y} a\right)\right)}},
$$

where $f_{0}$ is a resonant frequency of an individual element. Thus it can be seen that the upper cutoff frequency is $f_{0} \sqrt{\frac{1+\left|\kappa_{E}\right|}{1-\left|\kappa_{H}\right|}}$ and the lower one is $f_{0} \sqrt{\frac{1-\left|\kappa_{E}\right|}{1+\left|\kappa_{H}\right|}}$. For stronger magnetic and electric couplings between the elements, the wider will be the operational band of the metamaterial and hence, to obtain a broadband negative-index metamaterial one should use a structure with both strong and negative electric and magnetic couplings between elements.

Using the coupling retrieval method discussed in [23], it can be demonstrated that helical elements in a planar arrangement have both negative magnetic and electric coupling between them. This results in negative dispersion as demonstrated in [10]. It can also be shown that by introduction of metallic caps on the top and bottom of the helices it is possible to obtain enhanced negative electric coupling while introducing holes in those caps allows one to largely conserve the negative magnetic coupling [24].

In this work we explore structures of such capped helices that combine negative magnetic and electric couplings. As a result, this strongly subwavelength structure provides an extremely broad negative-index mode that provides, to the best of our knowledge, a significantly wider percentage bandwidth at low GHZ frequency range than those presented in the literature. In Sec. II the numerical modeling method will be discussed and results for both square and hexagonal arrays of capped helices as well as helical structures arranged on a ground plane will be demonstrated. Section III concerns the manufacture of one specific hexagonal structure while the experimental setup to obtain its dispersion is presented in Sec. IV. Experimental results obtained for the manufactured geometry are presented in Sec. V. Ways to enhance the

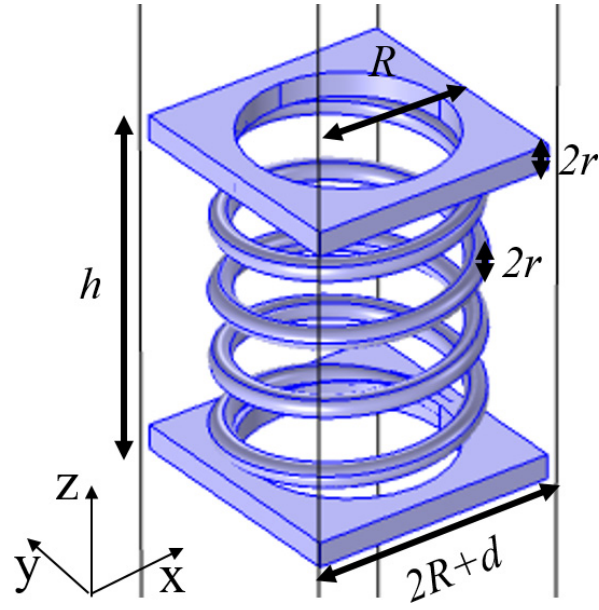

FIG. 1. Geometry used in COMSOL for the double capped helices square array unit cell. $l$ is the length of the helix, its outer radius is $R \mathrm{~mm}$, and the helix wire radius is $r$.

operational band even further will first be discussed in Sec. VI and the higher order modes of capped helices structures will be demonstrated in Sec. VII. Finally, the conclusions and possible applications of such structures are presented in Sec. VIII.

\section{NUMERICAL MODELING OF CAPPED HELICES ARRAYS}

\section{A. Numerical modeling method}

The finite element method was implemented using the eigenfrequency solver of the commercial package COMSOL Multiphysics in order to conduct numerical modeling of the studied structures. As structures discussed in this work are periodic, Floquet-periodic boundary conditions have been used to simulate an infinite 2D array. The resistivity used for the structures is that of the titanium alloy $(\mathrm{Ti} 6 \mathrm{Al}-4 \mathrm{~V})$ used in the fabrication of the experimental sample, $\rho=1.12 \pm$ $0.02 \mu \Omega \mathrm{m}$. The surrounding material was defined as air.

\section{B. Square array of capped helices}

To begin with, let us consider the square array of capped helices discussed in the Introduction with the following parameters: number of turns $n=4.5$, height $h=3 \mathrm{~mm}$, outer radius $R=1.1 \mathrm{~mm}$, wire radius $r=0.1 \mathrm{~mm}$, cap thickness $2 r=0.2 \mathrm{~mm}$, radius of hole in the cap $r_{\mathrm{h}}=R-2 r=$ $0.8 \mathrm{~mm}$, and distance between caps edges $d=0.1 \mathrm{~mm}$. The geometry of one unit cell is presented in Fig. 1 with all the parameters marked. Applying the coupling retrieval method described in [23] to such elements, the values of the electric and magnetic coupling coefficients are found to be $\kappa_{E}=$ -0.58 and $\kappa_{H}=-0.29$, respectively. The resonant frequency of an individual element in this case is $f_{0}=4.3 \mathrm{GHz}$.

First consider only the lowest order mode of this structure. The dispersion of this mode along the $x$ axis is shown in Fig. 2 . The values of $k$ are normalized to the Brillouin zone boundary $k_{\mathrm{b}}$ equal to it is important to note that the forward mode at low frequencies does not lie on the free-space light line (blue line) but as if it were in a material with refractive index of 


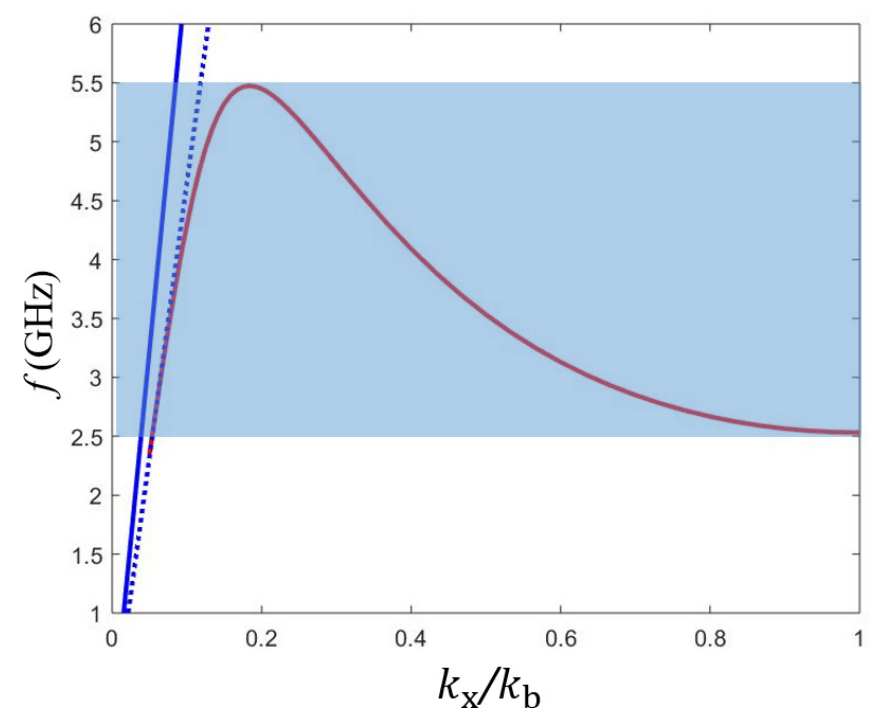

FIG. 2. Red solid line: Numerical (COMSOL) results for the dispersion of the eigenmodes of the square array of double capped helices for waves propagating along the $x$ axis. Blue solid line: Free-space light line. Blue dashed line: Light line in a material with refractive index of 1.35. Light blue shaded area marks the negative dispersion band. Unit cell shown in Fig. 1.

approximately 1.35 (blue dashed line). This effect arises from the polarizability of the individual helices.

The dispersion of the lowest frequency mode on this structure have been calculated analytically using Eq. (2) and is illustrated in Fig. 3. As the analytical model does not take into account the interaction with the "light line" (which in this case

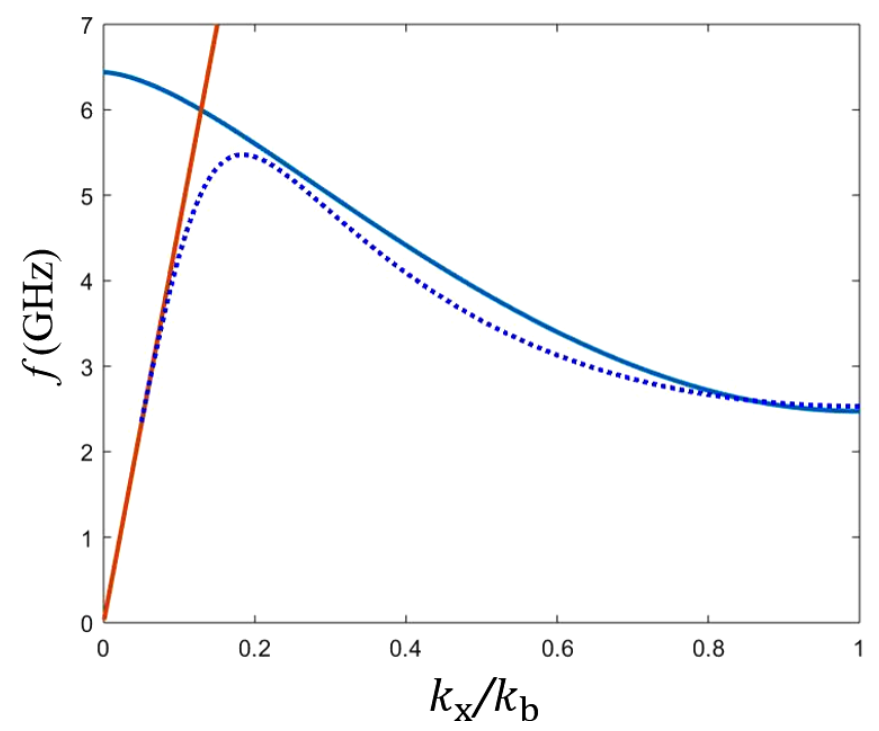

FIG. 3. Blue solid line: Analytical results for the dispersion of the eigenmodes of the square array of double capped helices for waves propagating along the $x$ axis obtained using Eq. (2) with $\kappa_{E}=0.58, \kappa_{H}=-0.29, f_{0}=4.3 \mathrm{GHz}$. Blue dotted line: Numerical (COMSOL) results for the dispersion of the eigenmodes of the square array of double capped helices for waves propagating along the $x$ axis. Red solid line: Light line in a material with refractive index of 1.35 .

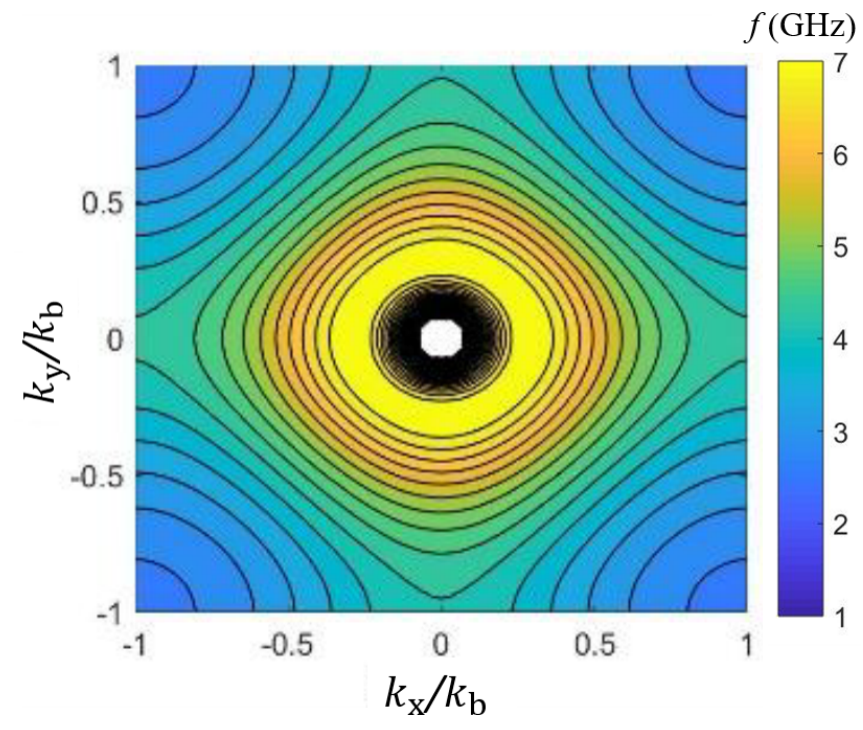

FIG. 4. Numerical results obtained for the isofrequency contours of the lowest order mode of the square array of capped helices. Unit cell shown in Fig. 1.

can be treated as an electromagnetic wave propagating in the structure with a refractive index of 1.35) the negative-index mode propagates between 2.47 and $6.43 \mathrm{GHz}$ unperturbed. However in the COMSOL model an anticrossing can be clearly seen between a negative-index mode and the light line mode. Good agreement between analytical and numerical data is observed for $k>0.2 k_{\mathrm{b}}$, where the dispersion is not affected by the anticrossing.

Note that for every frequency within the operational band (shaded) there are now two allowed $k$ values, thus both modes will be excited. (Later we discuss how this may be avoided.) Now the operational band, where the negative mode index is observed, is narrower than in the analytical case, from 2.5 to $5.4 \mathrm{GHz}$, and comprises $64 \%$ of the central frequency of operation. The small difference between the lowest frequencies can be explained by the approximations of the LCR circuit model while the highest frequency for numerical results is affected by the anticrossing.

While this structure provides a much wider operational band than those found in the literature, the dispersion is strongly anisotropic as is seen from the isofrequency contours numerically obtained for the lowest mode of this structure (Fig. 4). For many practical applications it may be useful to have structures with a more isotropic response. To address this issue we propose using an hexagonal array instead of a square one.

\section{Hexagonal array of capped helices}

A single element of the hexagonal array consisting of helices with hexagonal caps is shown in Fig. 5. The radii of the helix and the hole in the cap, the wire and cap thickness, and distance between cap edges are the same as the ones of the square structure.

Numerical results for the isofrequency contours of the lowest mode are illustrated in Fig. 6. For much of the operational band, a near isotropic response is observed. However, in the 


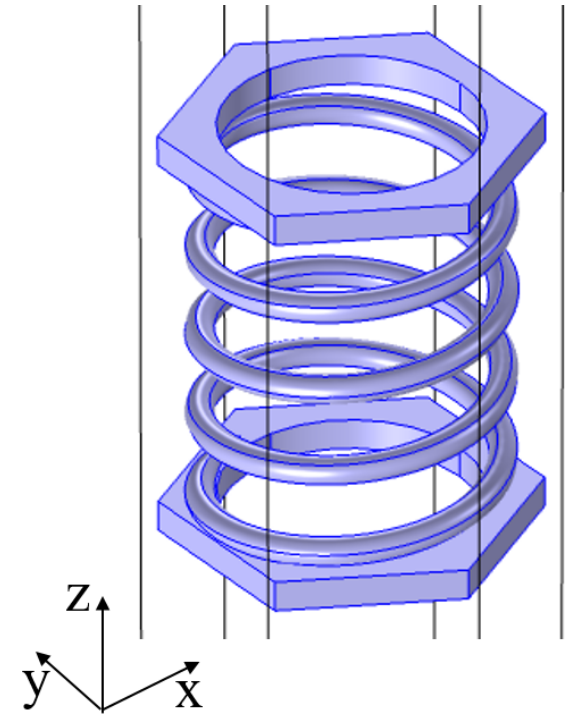

FIG. 5. Geometry used in COMSOL for the double capped helices in an hexagonal array.

low frequency, high wave-vector, region the isofrequency contours have hexagonal symmetry with the cutoff along the $x$ axis being lower in frequency than that for the $y$ axis. Comparison between the dispersions along $x$ and $y$ axes are shown in Fig. 7. The operational band of this structure is $80 \%$, which is larger than that for the square array due to the fact that each element is strongly coupled to six neighboring elements compared to only four in the case of the square array. However, there are several ways to increase the coupling values and thus broaden the bandwidth of the negative dispersion region even further. (Higher order modes in this case have much the same overall dispersion structure as for the square array.) Here and further on we will calculate the operational band of the hexagonal structures based upon the lowest and highest frequencies in the $y$ direction.

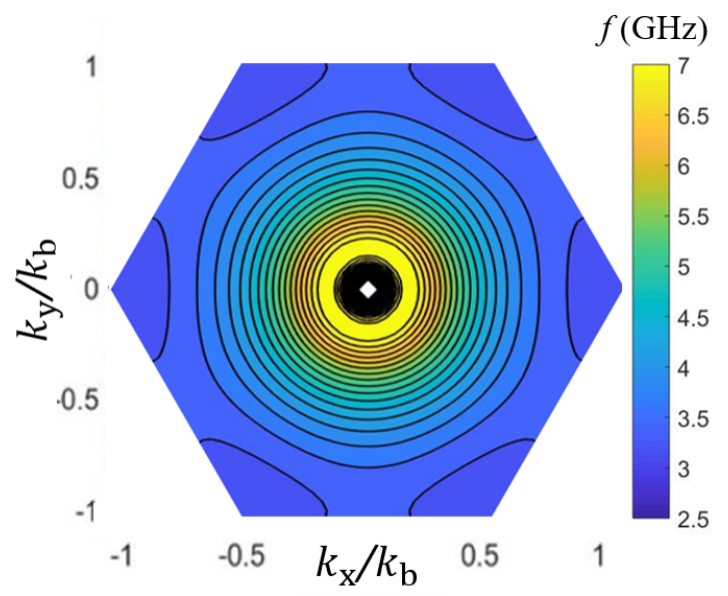

FIG. 6. Numerical results obtained for the isofrequency contours of the lowest order mode of the hexagonal array of capped helices. The element geometry is shown in Fig. 5.

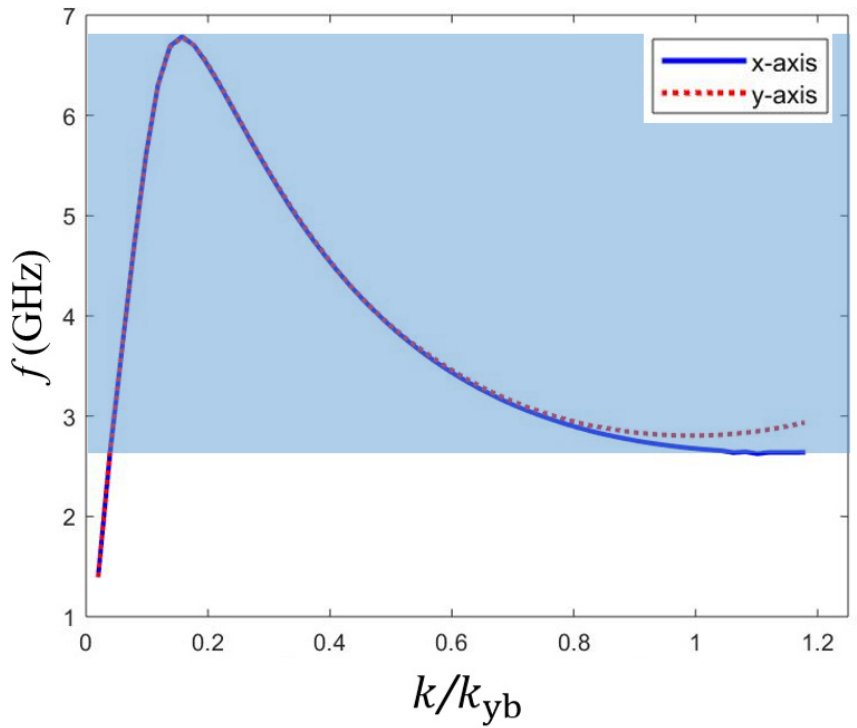

FIG. 7. Numerical results for the eigenfrequencies of the lowest order mode of the double capped helices hexagonal array with the wave propagating along $x$ (blue) and $y$ (red) axes.

\section{Hexagonal array of capped helices on a ground plane}

One way to simplify the manufacture of these capped helix structures is to arrange the elements on a ground plane. The element geometry of such an hexagonal structure is shown in Fig. 8 in the inset. Now only one end of the helices is capped, the ground plane effectively mirrors the fields of the capped helix on top of it, thus largely preserving the broadband negative-index response while shifting the operational frequency down by effectively doubling the length of the helices. The dispersion of the lowest mode for such a structure along the $y$ axis is illustrated in Fig. 8. It provides a $72 \%$

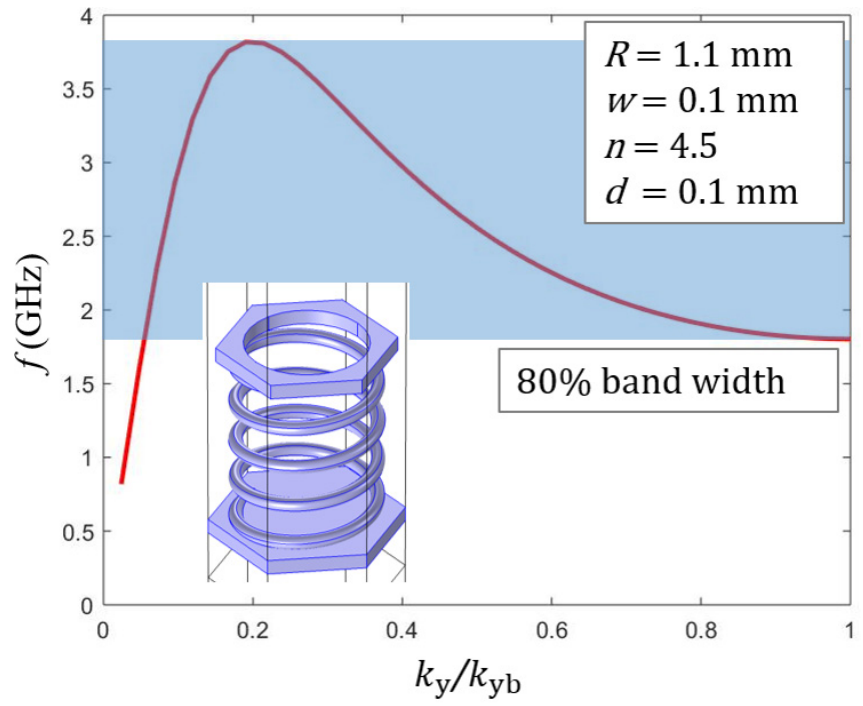

FIG. 8. Numerical results for dispersion along the $x$ axis of the modes of the hexagonal array of capped helices on a ground plane. Inset: Corresponding element geometry used in COMSOL, geometrical parameters of the structure and operational bandwidth. 
(a)

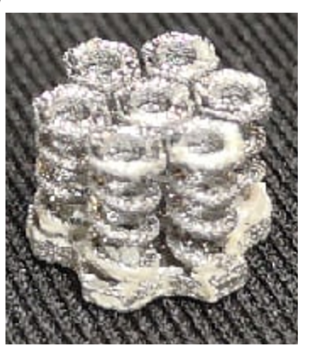

(b)

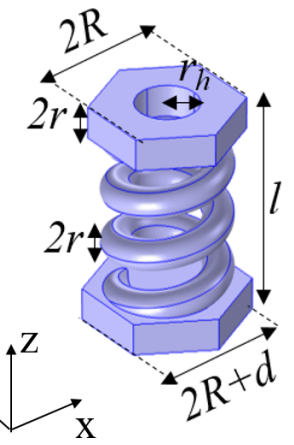

FIG. 9. (a) Photograph of the three-dimensional printed seven capped helix element on a ground plane. (b) COMSOL geometry used to model the hexagonal array of such capped helices.

operational band with $2.8 \mathrm{GHz}$ central frequency. Although it is narrower than for the double capped helix with the same parameters, it is still significantly wider than previously suggested structures.

\section{MANUFACTURING}

We fabricate the discussed structure by additive manufacturing using laser powder bed fusion (L-PBF) of a titanium alloy (Ti 6Al-4V). Samples were manufactured using a Renishaw AM125 L-PBF machine. The resistivity of the printed structure $\rho=1.12 \pm 0.02 \mu \Omega \mathrm{m}$ was determined based on measuring a $30 \times 1 \times 1 \mathrm{~mm}$ bar built using the same L-PBF process and material. Helices were manufactured in groups of seven in an hexagonal arrangement as shown in Fig. 9(a), and then assembled into the 2D array. Parameters of the helices as given in Fig. 9(b) are: $R=1.3 \mathrm{~mm}$, radius of holes $r_{h}=0.6 \mathrm{~mm}, r=0.33 \mathrm{~mm}, l=4.5 \mathrm{~mm}, n=3.5$. Helices have been arranged on the ground plane so that the distance between the top caps is $d=0.2 \mathrm{~mm}$.

These parameters have been chosen to satisfy the manufacturing capabilities in terms of printing resolution. Due to practical restrictions in terms of cost and time, only a limited number of elements were printed (117 of the presented geometry), the size of resulting surface is limited to about $100 \times 100 \mathrm{~mm}^{2}$. Due to this finite size, the helix geometry was designed so that the operational band was above $3 \mathrm{GHz}$ to allow experimental measurement of surface-wave dispersion.

\section{EXPERIMENTAL SETUP}

In order to experimentally determine the eigenmodes of the structure, we have arranged the seven helix elements in an overall approximately rectangular shaped area of 86 elements, with seven capped helices each [shown in Fig. 10(a)]. Here and further on we will keep the $x$ and $y$ axes associated with the geometry of an individual capped helix shown in Fig. 8. Instantaneous electric field profiles of the surface-waves that are excited from a point-probe source located at the edge of the sample have been measured using an electric near-field probe positioned $0.2 \mathrm{~mm}$ above the top of the caps for the frequency range 2 to $6 \mathrm{GHz}$. For this purpose we have used a stripped coaxial cable with outer radius of $1 \mathrm{~mm}$, inner wire radius $0.1 \mathrm{~mm}$ with a stripped part length of $2 \mathrm{~mm}$. Positioning of the probes was controlled to a precision of $0.1 \mathrm{~mm}$ using a computer controlled $x y z$-translation stage. The probes are connected to port 1 and port 2 of a vector network analyser (Anritsu MS4644A). A Fourier transform is then performed upon the spatial measured electric field distribution, with the magnitude of the Fourier terms indicating the strength of the wave vector components present in the spatial field measurements.

To obtain the dispersion in $x$ and $y$ directions, respectively, feeding probes have been positioned as shown in Figs. 10(a) and $10(\mathrm{~b})$, respectively. Scans have been taken along the marked lines with $1 \mathrm{~mm}$ steps across both axes. Then the data from nine linear scans have been averaged and the Fourier

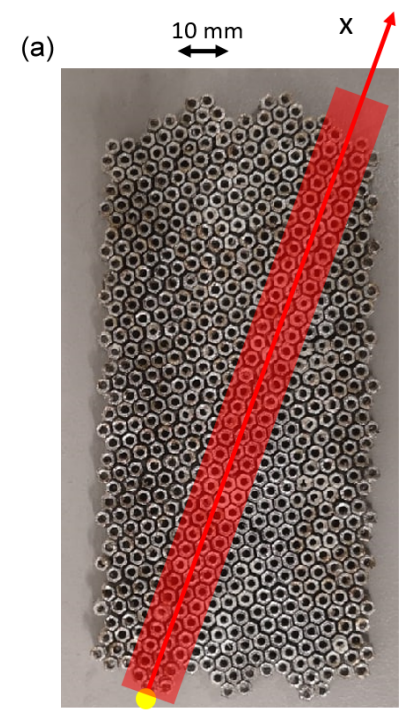

(b)

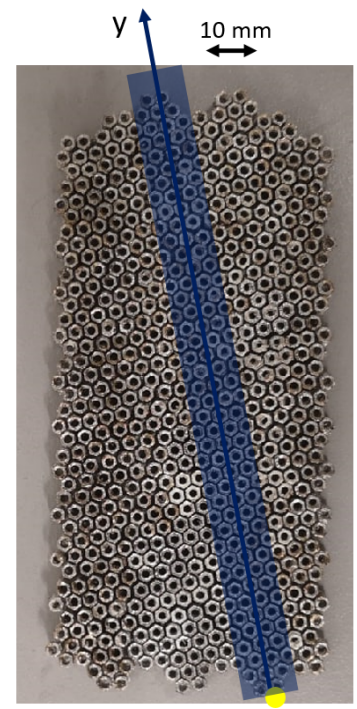

(c)

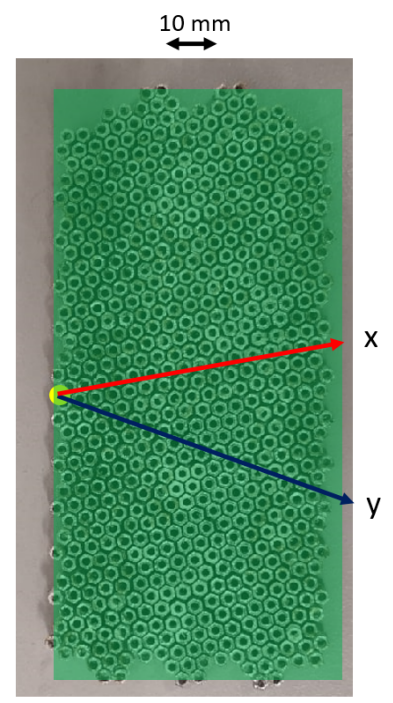

FIG. 10. Photograph of an assembled hexagonal array of three-dimensional printed capped helices on the ground plane. Yellow circles mark the microwave feeding points, shaded areas mark the area where scans have been taken for each particular case. (a) Measurement of the waves propagating along the $x$ axis. (b) Measurement of the waves propagating along the $y$ axis. (c) 2D scan of the surface. 

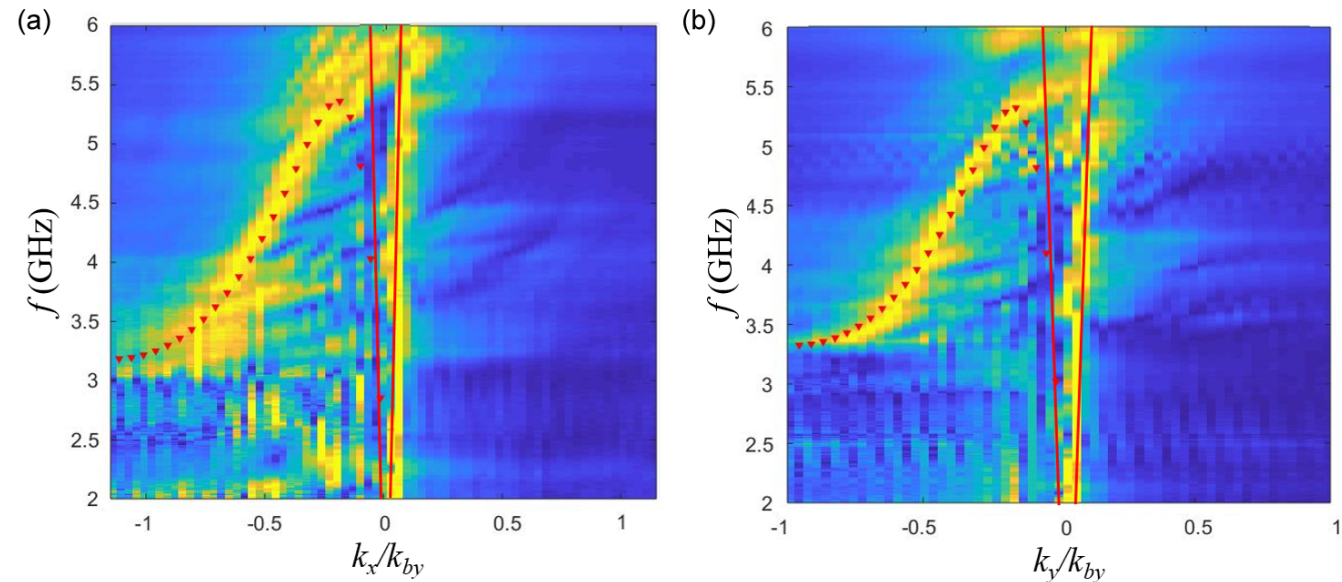

FIG. 11. Experimental and numerical (red triangles) results for eigenfrequencies of the lowest order mode of the capped helices hexagonal array on the ground plane with a wave propagating along (a) $x$ and (b) $y$ axes. Red solid line represents the light line. The element geometry is shown in Fig. 9.

transform of the resulting data has been obtained. To obtain the isofrequency contours of this structure, the probe has been positioned as marked in Fig. 10(c), and a two-dimensional scan with $1 \mathrm{~mm}$ steps in both directions has been done over the whole surface. 2D Fourier transform has then been applied to this data.

\section{EXPERIMENTAL RESULTS}

Experimental and numerical results for the dispersion of this structure along the $x$ and $y$ directions are shown in Figs. 11(a) and 11(b). To obtain these plots the amplitudes of the Fourier coefficients have been normalized at each frequency. After that, the normalization has been done for all the $k$ vectors, thus the local maxima of Fourier transform at each frequency and each $k$ vector, which correspond to eigenfrequencies of the system, will be equal to one. Finally, to improve the contrast of the negative-index mode compared to the light line, the area of negative $k$ values beyond the light line has been normalized separately. The good agreement between numerical and experimental results can be seen in both directions. As expected, the cutoff along the $x$ axis is at a lower frequency $(3.2 \mathrm{GHz})$ compared to the one for the $y$ axis $(3.35 \mathrm{GHz})$. An operational band of $49 \%$ has been obtained for this structure, which is to the best of our knowledge, almost 2.5 times larger than any reported in the literature. As the sample has been excited from the edge only, negative $k$ values (corresponding to the forward power transfer) can be observed for the "backward wave."

The experimental results for the isofrequency contour at 4.5 $\mathrm{GHz}$ are illustrated in Fig. 12. Here the region with $k$ values smaller than the ones of a free-space electromagnetic wave $\left(k_{0}\right)$ has been removed to increase the contrast of the negative-index mode. Asymmetry in the plot can be explained by the fact that the axis of the lattice does not coincide with the axis of the sample and the fact that the sample is fed from the edge which results in negative-index waves being observed only in the negative half of the $k$ space. It can be seen that the response is isotropic for the allowed $k$ values.

\section{BANDWIDTH OPTIMIZATION}

Although we have thus far been limited by manufacturing capabilities, it is informative to explore how the operational band of such structures can be improved even further. According to Eq. (2), to increase the operational bandwidth one should increase the value of coupling between elements in the array. For the following discussion we will use the manufactured structure as the initial one, and make certain changes relative to it with all other parameters of the discussed structures assumed to be the same as in the initial one.

The easiest way to increase the electric, and to a limited extent the magnetic coupling, is to reduce the distance between caps edges. Numerical results for the eigenfrequencies of the first mode of the hexagonal array with $d=0.1 \mathrm{~mm}$ is shown in Fig. 13(a) with the geometry illustrated in the inset. The operational band is now $63 \%$ of the central frequency between 1.95 and $3.75 \mathrm{GHz}$, which is significantly larger compared to $49 \%$ for the initial structure.

One way to strengthen the magnetic coupling is to increase the diameter of the hole in the middle of the caps relative

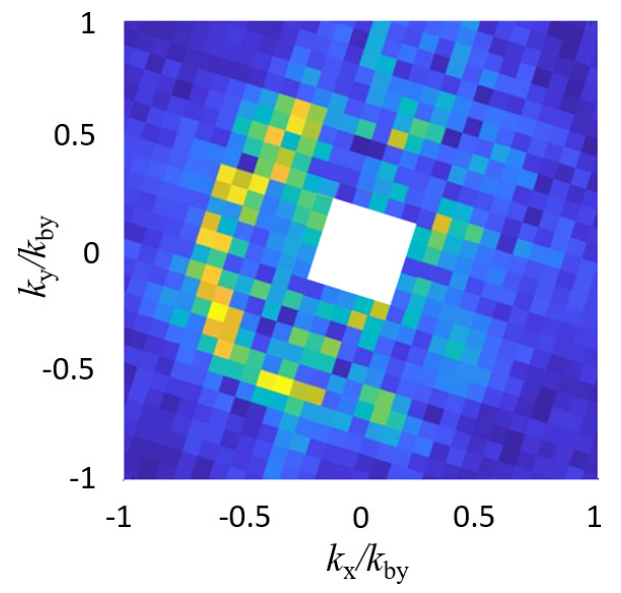

FIG. 12. Experimental results obtained for the isofrequency contours of the hexagonal array dispersion at $4.50 \mathrm{GHz}$. Data with $k<k_{0}$ has been removed. The element geometry is shown in Fig. 9. 

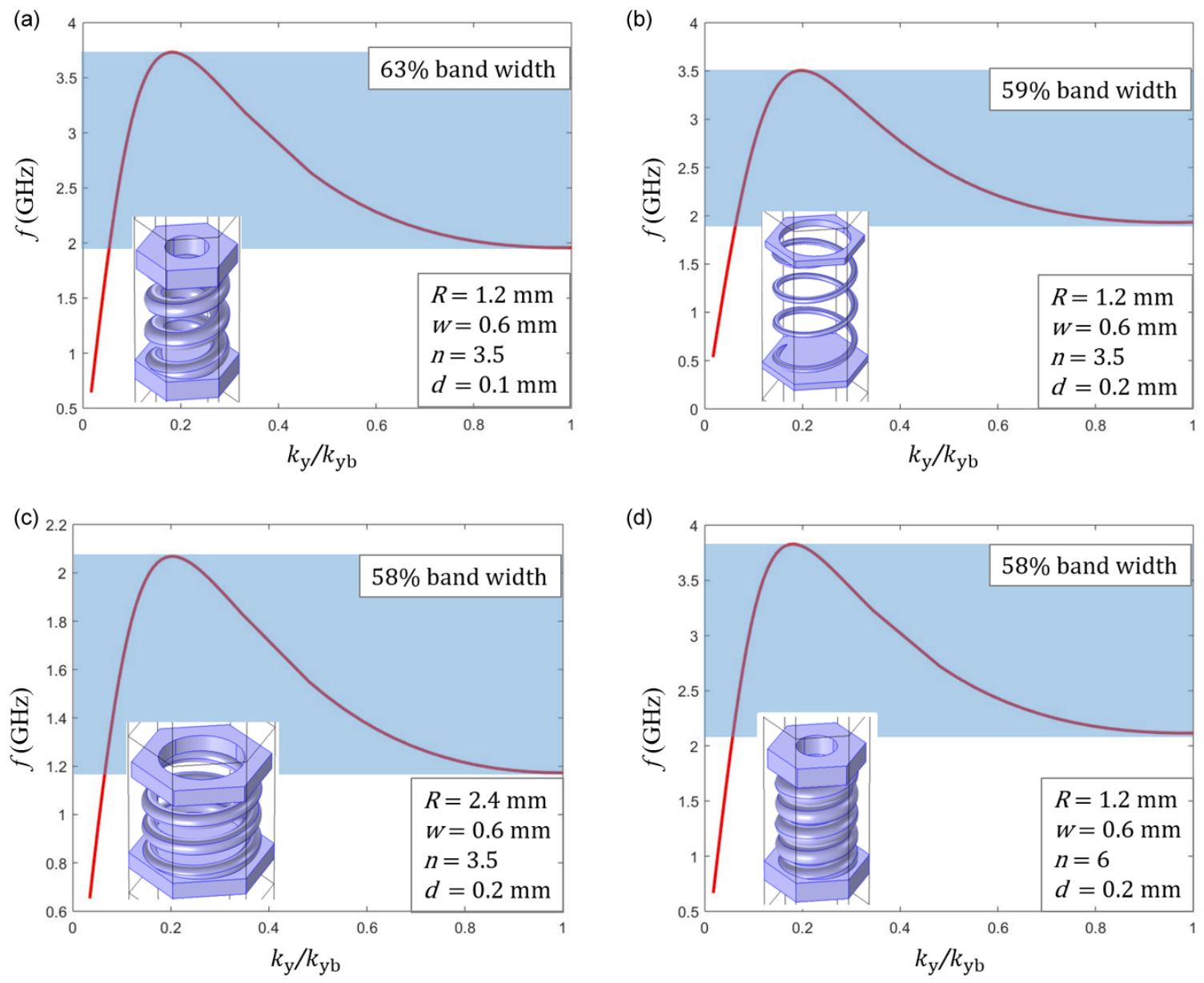

FIG. 13. Numerical results for eigenfrequencies of the lowest order mode of the capped helices hexagonal arrays on the ground plane with wave propagating along the $y$ axis. Inset: Corresponding element geometry used in COMSOL, their geometrical parameters and operational bandwidth.

to the outer helix diameter. This can be done using several approaches. First, the helix wire and cap can be made thinner. For instance, the performance of the hexagonal structure with the same parameters as the experimentally realized one but with $w=0.1 \mathrm{~mm}$ and $r_{h}=1 \mathrm{~mm}$ is shown in Fig. 13(b) with the geometry illustrated in the inset. The operational band is now $59 \%$ of the central frequency between 1.93 and $3.5 \mathrm{GHz}$ that is $10 \%$ larger than that of the initial structure.

The second approach to magnetic coupling improvement is to increase the radius of the whole structure. For instance, using an hexagonal array of capped helices with $R=2.6 \mathrm{~mm}$, $r_{h}=1.9 \mathrm{~mm}$, with all other parameters the same as in the experimental geometry, as shown in Fig. 13(c). Note that periodicity of the unit cell here is changed accordingly so that the gap between elements is $d=0.2 \mathrm{~mm}$ as in the initial case. It provides an operational band similar to the thin wire structure $(58 \%)$ but at significantly lower frequencies between 1.18 and $2.1 \mathrm{GHz}$. Although the helix radius can be increased almost indefinitely, the magnetic coupling quickly saturates approaching the values for uncapped helices and further increase of the helix diameter will only make the structure more subwavelength in the $z$ direction. Similarly infinitesimally thin helix wires will not improve the operational band much further.
There is also another way to strengthen the electric coupling by increasing the number of turns in the helix. As an example we increase the number of turns to six for the experimentally realized hexagonal structure [geometry shown in Fig. 13(d)]. This also reduces the resonant frequency of the individual helices making them more subwavelength. The operational band of the structure now lies between 2.09 and $3.82 \mathrm{GHz}$ and again comprises $58 \%$ of the central frequency.

The discussed approaches can be easily combined as shown in Fig. 14. The hexagonal array of capped six turn helices with $R=2.6 \mathrm{~mm}, w=0.1 \mathrm{~mm}$, and $d=0.1 \mathrm{~mm}$ allows one to obtain an operational band of $100 \%$ with a central frequency of $0.75 \mathrm{GHz}$. However optimizing the structure like this makes it more complex and, as mentioned before, introduces almost insurmountable manufacturing difficulties.

\section{HIGHER ORDER MODES}

Now we turn attention to the higher order modes of these structures. We will again start from the double capped helices in the hexagonal array shown in Fig. 5. Numerical results for the dispersion of this array up to $20 \mathrm{GHz}$ are shown in Fig. 15. The lowest order mode (marked 1), which has been described in Sec. II C (Fig. 7), is separated from higher order 


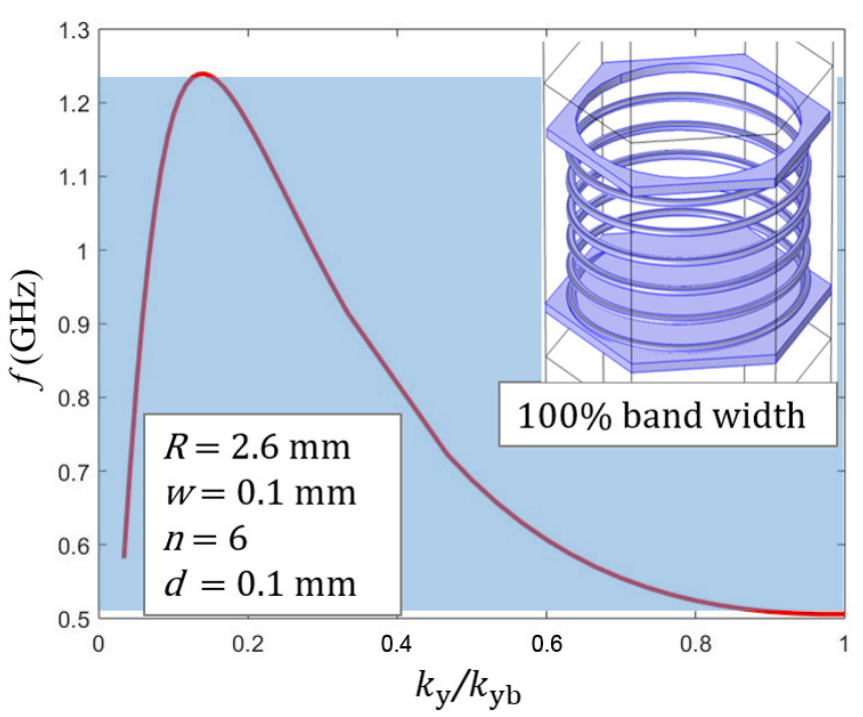

FIG. 14. Numerical results for eigenfrequencies of the lowest order mode of the capped helices hexagonal array on the ground plane with wave propagating along the $y$ axis. Inset: Element geometry used in COMSOL.

ones by a $2 \mathrm{GHz}$ stop band where no waves are supported. Two modes, degenerate at $10 \mathrm{GHz}(2$ and 3 in Fig. 15) are orthogonal to each other with the lower being coupled to the first mode of the structure with $\boldsymbol{H}$ field being mainly tangential to the direction of propagation, and $\boldsymbol{E}$ parallel to the $z$ axis [transverse magnetic (TM)]. On the other hand, for the second mode the $\boldsymbol{E}$ field is tangential to $\boldsymbol{k}$ and $\boldsymbol{H}$ is orthogonal to it [transverse electric (TE)]. Mode 3', although being a continuation of mode 3 , is a result of the anticrossing with mode 5 starting at $18 \mathrm{GHz}$. It is associated with the second order resonance of the helices and, similar to the lowest mode, it has

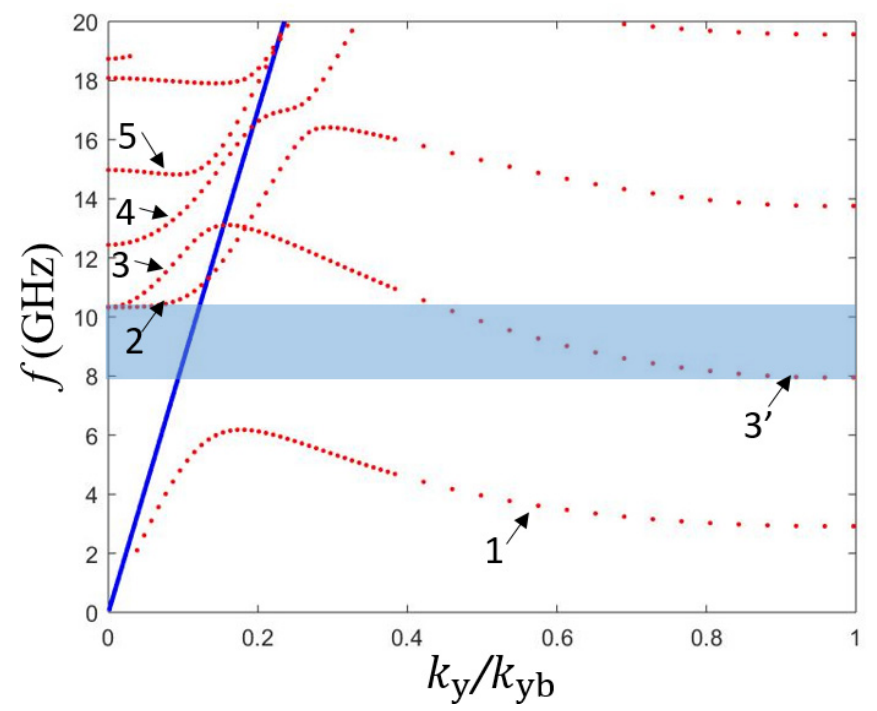

FIG. 15. Red dots: Numerical results for the dispersion of the eigenmodes of the square array of double capped helices for waves propagating along the $x$ axis. Blue solid line: Free-space light line. Light blue shaded area marks the negative dispersion band where only one mode propagates. Unit cell shown in Fig. 5.

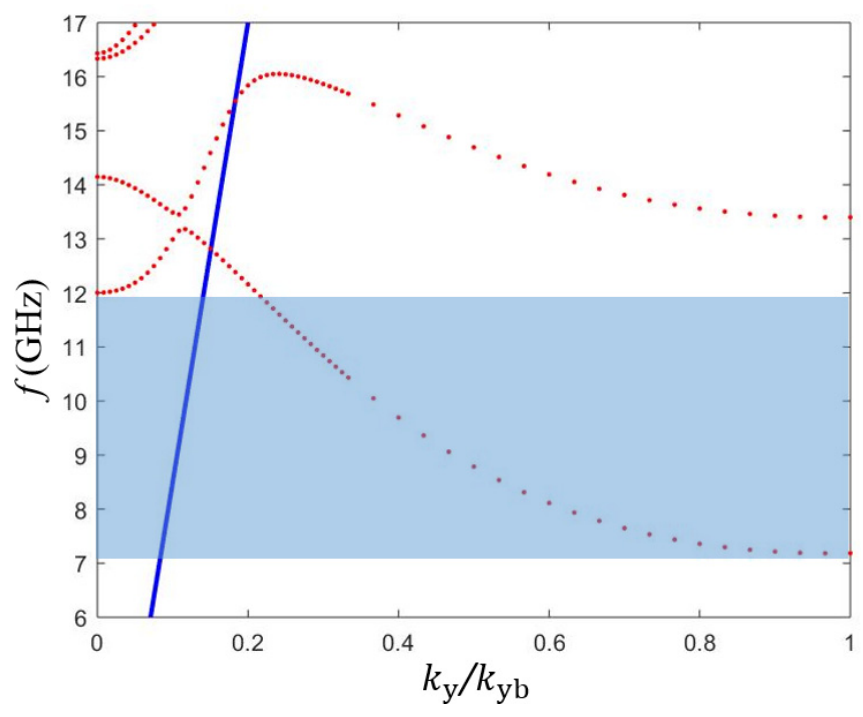

FIG. 16. Red dots: Numerical results for dispersion of the eigenfrequencies of dispersion modes of the capped helices hexagonal array on a ground plane for the wave propagating along the $x$ axis. Blue solid line: Free-space light line. Light blue shaded area mark the corresponding negative dispersion band where only one mode propagates. Unit cell shown in Fig. 8.

negative dispersion. Higher order modes corresponding to the higher order resonances of the helices may also have negative dispersion. The outstanding feature of the second order mode is that over a narrow frequency band between 8 and $10.2 \mathrm{GHz}$ only the negative-index mode is excited and thus, over this bandwidth, no conventional forward waves are supported by this structure. (This effect is not observed for the lowest mode as the forward wave propagating along the light line cannot be avoided below the first resonance of the structure.) Such a

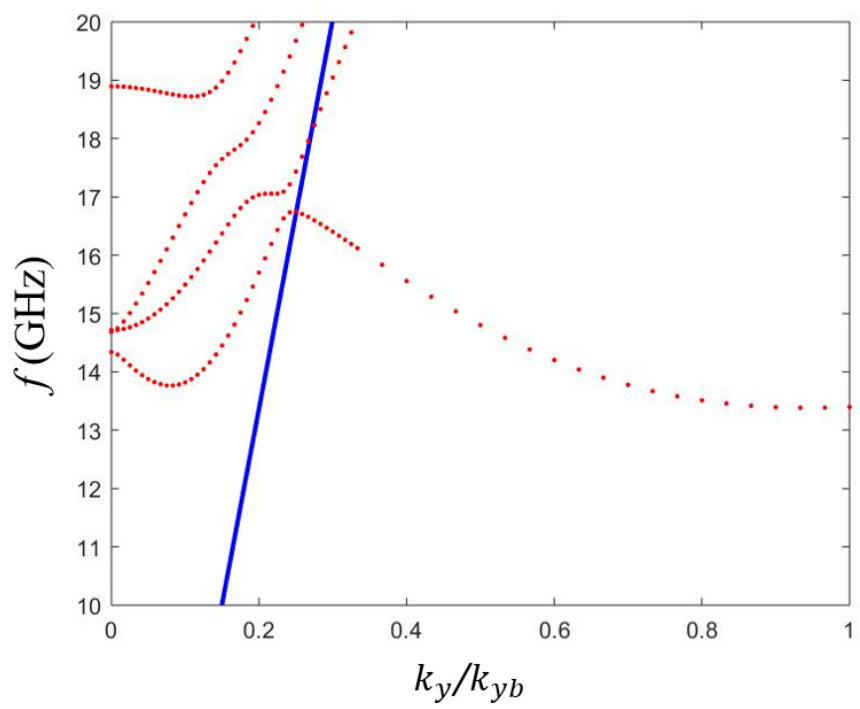

FIG. 17. Red dots: Numerical results for eigenfrequencies of dispersion modes of the capped helices hexagonal array on the ground plane with wave propagating along the $x$ axis. Blue solid line: Freespace light line. Unit cell shown in Fig. 9. 


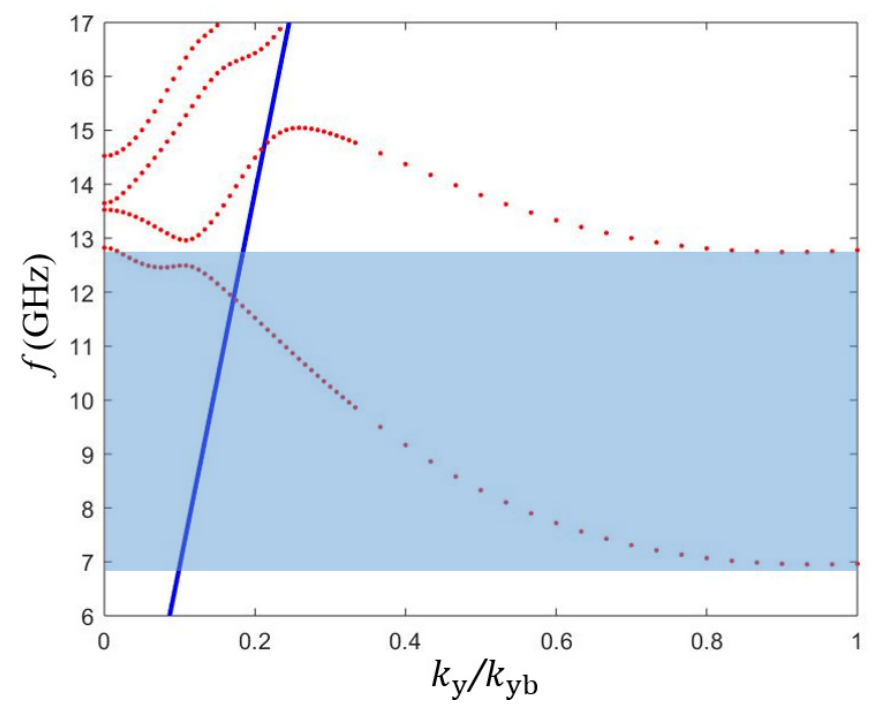

FIG. 18. Red dots: Numerical results for eigenfrequencies of dispersion modes of the capped helices hexagonal array on the ground plane with wave propagating along the $y$ axis. $w=0.1 \mathrm{~mm}$, $r_{h}=1 \mathrm{~mm}$. Blue solid line: Light line. Light blue shaded area marks the corresponding negative dispersion band where only one mode propagates. Unit cell shown in Fig. 13(b).

mode, although having a narrower operational band than the first one, may have applications in microwave imaging.

If once more we use an hexagonal array and a ground plane as introduced in Fig. 8, mode "2," observed for the previous structure, is no longer supported, as demonstrated in Fig. 16. Furthermore, the higher order modes are shifted to higher frequencies relative to the negative index modes. As a result, the second negative-index mode has only one anticrossing at 13.2 GHz and, more importantly, the operational band where no conventional forward waves are supported is now between between 7.1 and $12 \mathrm{GHz}$ (52\% of its central frequency) that is already closer to the values obtained for the first negativeindex mode.

The frequencies of positive and negative-index modes in these structures are strongly dependent on the geometry of the helices and need to be optimized separately from coupling. For instance, the numerical results for dispersion of the higher order modes using the manufactured geometry are shown in Fig. 17. As can be seen, several modes inside the light line are interacting with the second negative-index mode and now there is no band where only that mode in excited.

All the structures studied above in this section have the positive-index modes which overlap with some part of the negative-index one which limits the frequency band where only backward wave is supported. This effect is observed for most of the structures, however for the the geometry shown in Fig. 13(b), the positive-index mode is shifted to higher frequencies and has minimum overlap with the second negative-index mode. Numerical results for the higher order dispersion modes of this structure are presented in Fig. 18. Two eigenfrequencies can be observed at zero $k$ at 12.81 and $13.62 \mathrm{GHz}$, respectively. The lower one corresponds to the anticrossing of the first mode with the light line mode, and the higher one is part of the negative-index mode corresponding to the second order resonance of the helices. Therefore, it is not possible to obtain a geometry with the former eigenfrequency being at a higher frequency than the latter. However, with this configuration, with the anticrossing between modes occurring at a low $k$ value, a negative dispersion curve is found between 6.96 and $12.81 \mathrm{GHz}$ without any region where both positiveand negative-index modes are observed. This provides an operational band of $58 \%$ similar to that of the first mode for this structure.

\section{CONCLUSIONS}

A metasurface comprised of capped helices that supports a broadband negative-index surface-wave has been manufactured using laser powder-bed-fusion additive manufacturing. The results for the mode dispersion of this structure are demonstrated numerically and experimentally with the operational band being between 3.4 and $5.4 \mathrm{GHz}$ or $49 \%$ of the central frequency. This is more than double the bandwidth of structures previously reported in the literature. Suggestions are offered for ways to widen the operational band even further, with a specific geometry offering $100 \%$ bandwidth being demonstrated.

It was also shown that such structures can be optimized to provide a negative-index operational band using the second order resonance of the helices, where no forward waves are simultaneously supported. Model results show a potential bandwidth of 59\% for this mode, although sample manufacturing difficulties may be substantial.

The structures discussed in this work broaden the range of material parameters that can be realized using metamaterials. The first negative-index mode of these structures can possibly be used for microwave power transfer and in waveguides, while the second order one can also open further possibilities for microwave imaging and perfect lenses.

The research data supporting this publication are openly available from the University of Exeter's institutional repository at [25].

\section{ACKNOWLEDGMENTS}

We acknowledge financial support from the Engineering and Physical Sciences Research Council (EPSRC) of the United Kingdom via the EPSRC Centre for Doctoral Training in Metamaterials (Grant No. EP/L015331/1). We also acknowledge financial support from the Defence Science and Technology Laboratory (DSTL) (Contract No. DSTLX1000133579).
[1] H. Lamb, Mapping inter-element coupling in metamaterials: Scaling down to infrared, Proc. London Math. Soc. s2-1, 473 (1904).

[2] A. Schuster, Mapping inter-element coupling in metamaterials: Scaling down to infrared, An introduction to the theory of optics, 330 (1904), https://archive.org/details/bub_ gb_Zb4KAAAAIAAJ.

[3] V. G. Veselago, The electrodynamics of substances with simultaneously negative values of permittivity and permeability, Sov. Phys. Usp. 10, 509 (1968). 
[4] J. B. Pendry, A. J. Holdenz, D. J. Robbinsz, and W. J. Stewartz, Low frequency plasmons in thin-wire structures, J. Phys. Condens. Matter. 10, 4785 (1998).

[5] J. Pendry, A. Holden, D. Robbins, and W. Stewart, Magnetism from conductors and enhanced nonlinear phenomena, IEEE Trans. Microwave Theory Tech. 47, 2075 (1999).

[6] A. Wagner, C. Müntz, H. Oeschler, C. Strum, R. Barth, M. Cieślak, M. Dębowski, E. Grosse, P. Koczoń, F. Laue, M. Mang, D. Miśkowiec, E. Schwab, P. Senger, P. Beckerle, D. Brill, Y. Shin, H. Ströbele, W. Waluś, B. Kohlmeyer, F. Puhlhofer, J. Speer, and I. K. Yoo, Emission Pattern of High-Energy Pions: A New Probe for the Early Phase of Heavy-Ion Collisions, Phys. Rev. Lett. 85, 18 (2000).

[7] X.-G. He, C.-L. Hsueh, and J.-Q. Shi, Constraints on the Phase $\gamma$ and New Physics from $B \rightarrow K \pi$ Decays, Phys. Rev. Lett. 84, 18 (2000).

[8] R. A. Shelby, D. R. Smith, and S. Schultz, Experimental verification of a negative index of refraction, Science 292, 77 (2001).

[9] M. C. K. Wiltshire, J. B. Pendry, I. R. Young, D. J. Larkman, D. J. Gilderdale, and J. V. Hajnal, Microstructured magnetic materials for rf flux guides in magnetic resonance imaging, Science 291, 849 (2001).

[10] C. Wu, H. Li, Z. Wei, X. Yu, and C. T. Chan, Theory and Experimental Realization of Negative Refraction in a Metallic Helix Array, Phys. Rev. Lett. 105, 247401 (2010).

[11] U. K. Chettiar, A. V. Kildishev, H. K. Yuan, W. Cai, S. Xiao, V. P. Drachev, and V. M. Shalaev, Dual-band negative index metamaterial: Double negative at $813 \mathrm{~nm}$ and single negative at 772 nm, Opt. Lett. 32, 1671 (2007).

[12] J. Valentine, S. Zhang, T. Zentgraf, E. Ulin-Avila, D. A. Genov, G. Bartal, and X. Zhang, Three-dimensional optical metamaterial with a negative refractive index, Nature (London) 455, 376 (2008).

[13] D. Sievenpiper, L. Zhang, R. F. J. Broasand, N. G. Alexopolous, and E. Yablonovitch, High-impedance electromagnetic surfaces with a forbidden frequency band, IEEE Trans. Microwave Theory Tech. 47, 2059 (1999).
[14] J. A. Dockrey, S. A. R. Horsley, I. R. Hooper, J. R. Sambles, and A. P. Hibbins, Direct observation of negative-index microwave surface waves, Sci. Rep. 6, 22018 (2016).

[15] G. Bouchitt'e and B. Schweizer, Cloaking of small objects by anomalous localized resonance, Quart. J. Mech. Appl. Math. 63, 437 (2010).

[16] R. Yang and J. Wang, Broadband microwave negative index metamaterial with low dispersion, IEEE MTTS International Conference on Numerical Electromagnetic and Multiphysics Modeling and Optimization (NEMO) (IEEE, Piscataway, NJ, 2016).

[17] F. Ling, Z. Zhong, R. Huang, and B. Zhang, A broadband tunable terahertz negative refractive index metamaterial, Adv. Opt. Mater. 8, 9843 (2018).

[18] A. C. Atre, A. García-Etxarri, H. Alaeian, and J. A. Dionne, A broadband negative index metamaterial at optical frequencies, Adv. Opt. Mater. 1, 327 (2013).

[19] J. B. Pendry, Negative Refraction Makes a Perfect Lens, Phys. Rev. Lett. 85, 3966 (2000).

[20] S. Bang, S. So, and J. Rho, Realization of broadband negative refraction in the visible range using vertically stacked hyperbolic metamaterials, Sci. Rep. 9, 14093 (2019).

[21] A. Radkovskaya, M. Shamonin, C. J. Stevens, G. Faulkner, D. J. Edwards, E. Shamonina, and L. Solymar, Resonant frequencies of a combination of split rings: Experimental, analytical and numerical study, Microwave Opt. Technol. Lett. 46, 473 (2005).

[22] E. Tatartschuk, N. Gneiding, F. Hesmer, A. Radkovskaya, and E. Shamonina, Mapping inter-element coupling in metamaterials: Scaling down to infrared, J. Appl. Phys. 111, 094904 (2012).

[23] P. Petrov, A. Radkovskaya, and E. Shamonina, Retrieval of coupling coefficients for dense metamaterials, IEEE Proceedings of 10th International Congress on Advanced Electromagnetic Metamaterials in Microwaves and Optics (IEEE, Piscataway, NJ, 2016), p. 283.

[24] P. Petrov, A. P. Hibbins, and J. R. Sambles, Near-field electromagnetic coupling between helices, J. Phys. D: Appl. Phys. 54, 445108 (2021).

[25] See https://ore.exeter.ac.uk/repository/. 